\title{
Editorial: A Time of Transition of Alzheimer's Disease in the Advent of Anti-Amyloid Monoclonal Antibodies
}

Babak Tousi $\cdot$ Marwan Noel Sabbagh

Received: July 27, 2021 / Accepted: September 17, 2021 / Published online: October 13, 2021

(C) The Author(s) 2021

Keywords: Monoclonal

antibodies; Aducanumab; Disease-modifying treatments; Alzheimer's disease; Mild cognitive impairment

\section{EDITORIAL}

A substantial body of evidence supports the application of anti-amyloid monoclonal antibodies in the Alzheimer's disease (AD) clinical care setting, particularly in those with mild cognitive impairment and mild dementia. Monoclonal antibodies specifically target amyloid function by binding to amyloid beta $(A \beta)$ oligomers or other forms of amyloid (e.g., plaque) and clearing away the two proteins most strongly implicated in the development of $\mathrm{AD}$ and associated forms of dementia, $\mathrm{A} \beta$ and tau proteins, from neural cells. The monoclonal antibodies aducanumab, lecanemab, gantenerumab, and donanemab are of particular interest, and aducanumab has received Fast Track approval from the United States Food and Drug Administration. The approval was based more on the clearance of $A \beta$ from the brain by

\section{B. Tousi}

Cleveland Clinic Center for Brain Health, Cleveland, $\mathrm{OH}$, USA

M. N. Sabbagh $(\bowtie)$

Cleveland Clinic Lou Ruvo Center for Brain Health, 888 W. Bonneville Ave, Las Vegas, NV 89106, USA e-mail: marwan.sabbagh@dignityhealth.org aducanumab and less on the establishment of a clinical efficacy signal. This dichotomy has fueled controversy. Furthermore, monoclonal antibodies are showing promise for the development of new therapeutics targeting AD and associated forms of dementia. New therapies will need to fit into a new framework for patient care which takes into consideration protocols to mitigate the risk of virus exposure.

The COVID-19 pandemic has permanently altered patient care protocols, and new protocols are necessary for the implementation of monoclonal antibody therapy as well. From the presymptomatic stages to the confirmation of an AD diagnosis, a map clearly delineating the patient's journey must be constructed in light of post-COVID changes. Appropriate use guidelines have been proposed by which a patient can be accurately assessed and selected for anti-A $\beta$ monoclonal antibody treatments to ensure the highest possible quality of care at every step of the patient's journey, from suspicion of $\mathrm{AD}$ to a confirmed diagnosis [1].

Four anti-amyloid monoclonal antibodies are now in phase III or post-phase III clinical trials. These include aducanumab, lecanemab, gantenerumab, and donanemab [2]. All anti-A $\beta$ monoclonal antibodies have robust evidence of amyloid removal [3], and clinical data show evidence of slowing decline. Thus there is a strong possibility of one or more of these biologics being approved as a disease-modifying 
treatment for $\mathrm{AD}$ (mild cognitive impairment or Alzheimer's dementia).

With expectations increasing for the possibility of the first monoclonal antibody diseasemodifying treatment for $\mathrm{AD}$, there are concerns that physicians such as neurologists, primary care physicians, geriatricians, or psychiatrists are unable to effectively diagnose $\mathrm{AD}$, gather AD-directed biomarkers, or determine the appropriateness in patient selection for anti-A $\beta$ monoclonal antibodies.

\section{THE PATIENT'S JOURNEY, STEP BY STEP}

A patient's journey begins with a clinical presentation, based on the patient's and informant reports such as observations of someone close to the patient (e.g., a friend or family member), or genetic risk for $\mathrm{AD}$. At this stage, the patient may have already started manifesting the symptoms common to $\mathrm{AD}$, such as small lapses in memory or changes in mood or behavior. The clinical presentation of mild cognitive impairment includes a report of cognitive changes corroborated by an informant. The definition of mild cognitive impairment is a clinical one, with the phenotype including the absence of functional impairment [4]. In order to prepare patients for the era of disease-modifying treatments, the current evaluation process needs to evolve to add more steps.

\section{Defining the Clinical Phenotype}

The historical perspective for the determination of mild cognitive impairment is the presence of a cognitive change from premorbid functioning. This is amnestic in nature, without significant behavioral or personality changes. A suggested approach is outlined below.

First, the patient's cognitive changes are captured by informant-based questionnaires, such as the Eight-item Informant Interview to Differentiate Aging and Dementia (AD8), Informant Questionnaire on Cognitive Decline in the Elderly (IQCODE), and Alzheimer's Questionnaire (AQ) [5]. The AQ is an assessment with high sensitivity (greater than 95\%), in which a score greater than 10 indicates a high probability of Alzheimer's dementia or mild cognitive impairment [5]. Other questionnaires such as the Lewy Body Composite Risk Score [6] address specificity of screening for Lewy body symptoms. Validated questionnaires can be incorporated to exclude other common neurodegenerative conditions such as frontotemporal dementia or primary progressive aphasia.

The incorporation of aggregate risk analysis aids in the clinical diagnosis and increases the probability of AD. An aggregate score above 12 signals a high probability of the presence of $\mathrm{AD}$ [7].

Next, the patient undergoes bedside cognitive screening, such as the Montreal Cognitive Assessment (MoCA) [8] and Mini-Mental State Exam (MMSE) [9]. MoCA and MMSE scores below 25-26 indicate a higher probability of impairment. A thorough physical and neurological exam is then conducted to screen for normal pressure hydrocephalus, parkinsonism, or focal symptoms suggestive of cerebrovascular accident (CVA). Additional neuropsychological testing to determine the cognitive profile (amnestic versus non-amnestic) is an option.

The key change from standard practice is the diagnostic tools that are used. First, the patient might undergo neuropsychological evaluation to determine whether the clinical phenotype fits the Diagnostic and Statistical Manual of Mental Disorders, Fifth Edition (DSM-V) for mild neurocognitive disorder and the clinical profile of amnestic mild cognitive impairment. Amnestic mild cognitive impairment is defined as prodromal $\mathrm{AD}$ with higher risk of progression to $\mathrm{AD}$ dementia in most cases. In addition to neuropsychological testing, the evaluation should still include vitamin B12 and thyroidstimulating hormone testing to exclude B12 deficiency and hypothyroidism.

Magnetic resonance imaging (MRI) should be performed to determine white matter rarefaction, the presence of infarcts, the number of microhemorrhages, and the presence of communicating hydrocephalus or mass. NeuroQuant software can provide volumetric analysis by region and determine whether there is evidence of neurodegeneration [10]. 


\section{Identifying the Presence of Amyloid}

In order for patients to be eligible to receive the anti-amyloid disease-modifying treatments, confirmation of the presence of amyloid pathology will be needed. This can be derived in one of three ways. First, plasma $A \beta 42 / 40$ has Clinical Laboratory Improvement Amendments (CLIA) certification, with specificity and sensitivity of $75 \%$ [11]. At present, the test is not reimbursed by the United States Centers for Medicare \& Medicaid Services, but it is an appealing alternative because of the relatively noninvasive aspects. Second, amyloid positron emission tomography is approved by the United States Food and Drug Administration, and has shown very high sensitivity in detecting amyloid and altered patient management [12], but is not reimbursed by the US Centers for Medicare $\&$ Medicaid Services, thus limiting its usage. Third is cerebrospinal fluid (CSF) testing for A $\beta 42$ and total tau (t-tau) and phosphorylated tau (p-tau). The Elecsys platform has recently come into common use and detects the $A \beta 42 /$ 40 ratio, t-tau and p-tau, and the p-tau/amyloid ratio. It has good sensitivity but is invasive. Lumbar puncture and CSF analysis are covered by the US Centers for Medicare \& Medicaid Services. Although detection of amyloid will likely be required, it is not clear which modality will emerge, as each offers advantages and disadvantages. At present, it appears that CSF analysis is being ordered more often.

\section{Establishing the Diagnosis and Preparing for Infusion}

At this phase, if all other potential underlying conditions have been excluded, the probability of detecting $\mathrm{AD}$ is high in the setting of biomarker confirmation. By following these steps in this order and applying either International Working Group (IWG) criteria or National Institute on Aging-Alzheimer's Association (NIA-AA) working group criteria, the reliability of such a diagnosis can be ensured with high probability of accuracy. Eliminating underlying conditions that either accompany or mimic dementia can lead to more accurate detection of
$\mathrm{AD}$, thereby allowing therapeutic interventions, including newly emergent monoclonal antibody therapies, to commence in a timely and efficacious manner. Furthermore, comprehensive patient information would be essential for determining a patient's suitability for monoclonal antibody therapy and could be instrumental in calculating the probability of a more successful outcome for patients who opt for this particular type of therapeutic intervention.

\section{Monoclonal Antibody Treatments and Monitoring}

Infusion of the monoclonal antibodies aducanumab (monthly), lecanemab (twice monthly), and donanemab (monthly) will be per package insert. For gantenerumab (monthly in previous study and twice monthly in the GRADUATE study), the subcutaneous injection will also be per package insert. Post-approval, there will be protocols for monitoring for amyloid-related imaging abnormalities (ARIA) with serial MRI scans. ARIA is a term used to describe imaging abnormalities associated with anti-A $\beta$ monoclonal antibody therapy. They were noted as vasogenic edema or microhemorrhages after initial trials with the first group of monoclonal antibodies, and we continue to see these radiological changes with other monoclonal antibodies at different frequencies. ARIA can be subdivided into two groups: ARIA-E, which is parenchymal edema or sulcal edema detected on fluid-attenuated inversion recovery (FLAIR) images; and ARIA-H, which is hemosiderin deposition in the form of superficial siderosis or microhemorrhages as seen in gradient-recalled echo (GRE)/T2-weighted MRI.

There will be strategies for managing ARIA, since most are clinically asymptomatic and can be dosed through with some adjustments. It is not clear whether these protocols will be proscribed or physician-preferred. Ideally, a standardized reporting template should be used by the neuroradiologists in all centers to uniformly record radiological findings. Reports will be reviewed by clinicians prior to the next dosing increase to guide decisions about dosing continuation. 


\section{DISCUSSION}

The incorporation of anti-amyloid monoclonal antibodies as disease-modifying treatments for mild cognitive impairment due to $\mathrm{AD}$ will require some updating of the diagnostic approach. It will not be simply a description of the clinical phenotype and exclusion of other conditions [13]. Rather, it will be transformed into a more disease-specific approach, since up to $50 \%$ of mild cognitive impairment may not meet pathological criteria for $\mathrm{AD}$. Therefore, having biomarker evidence of $A \beta$ will be important for determining the suitability of monoclonal antibody treatment and for transforming $\mathrm{AD}$ clinically from a diagnosis of exclusion to a diagnosis of inclusion.

\section{ACKNOWLEDGEMENTS}

Funding. Supported by NIH COBRE 5P20GM109025, NIH P20 AG068053, NIH R01AG059008, and the Keep Memory Alive Foundation. No funding was received for the publication of this article.

Authorship. All named authors meet the International Committee of Medical Journal Editors (ICMJE) criteria for authorship for this article, take responsibility for the integrity of the work as a whole, and have given their approval for this version to be published.

Authors' Contributions. The content and ideas were co-developed and co-written by the authors.

Disclosures. Dr Babak Tousi discloses: Consulting: Esai, Biogen, Roche. Dr Marwan Noel Sabbagh discloses: Ownership interest (stock or stock options): Brain Health Inc, NeuroTau, Optimal Cognitive Health Company, uMethod Health, Versanum, Athira, Cognoptix. Consulting: Alzheon, Biogen, Cortexyme, Roche-Genentech, Stage 2 Innovations/Renew Research, Acadia, T3D, Eisai, KeifeRx. Dr Sabbagh's new affiliation is the
Barrow Neurological Institute, Phoenix, AZ, USA.

Compliance with Ethics Guidelines. This article is based on previously conducted studies and does not contain any new studies with human participants or animals performed by any of the authors.

Open Access. This article is licensed under a Creative Commons Attribution-NonCommercial 4.0 International License, which permits any non-commercial use, sharing, adaptation, distribution and reproduction in any medium or format, as long as you give appropriate credit to the original author(s) and the source, provide a link to the Creative Commons licence, and indicate if changes were made. The images or other third party material in this article are included in the article's Creative Commons licence, unless indicated otherwise in a credit line to the material. If material is not included in the article's Creative Commons licence and your intended use is not permitted by statutory regulation or exceeds the permitted use, you will need to obtain permission directly from the copyright holder. To view a copy of this licence, visit http://creativecommons.org/licenses/by$\mathrm{nc} / 4.0 /$.

\section{REFERENCES}

1. Cummings J, Aisen P, Apostolova LG, Atri A, Salloway S, Weiner M. Aducanumab: appropriate use recommendations; JPAD 2021, (published online).

2. Tolar M, Abushakra S, Hey JA, Porsteinsson A, Sabbagh M. Aducanumab, gantenerumab, BAN2401, and ALZ-801-the first wave of amyloid-targeting drugs for Alzheimer's disease with potential for near term approval. Alzheimer's Res Ther. 2020;12(1):95. https://doi.org/10.1186/s13195-020-00663-w.

3. Decourt B, Boumelhem F, Pope ED 3rd, Shi J, Mari Z, Sabbagh MN. Critical appraisal of amyloid lowering agents in AD. Curr Neurol Neurosci Rep. 2021;21(8):39. https://doi.org/10.1007/s11910-02101125-y.

4. Petersen RC. Mild cognitive impairment as a diagnostic entity. J Intern Med. 2004;256(3):183-94. https://doi.org/10.1111/j.1365-2796.2004.01388.x. 
5. Sabbagh MN, Malek-Ahmadi M, Kataria R, Belden CM, Connor DJ, Pearson C, Jacobson S, Davis K, Yaari R, Singh U. The Alzheimer's questionnaire: a proof of concept study for a new informant-based dementia assessment. J Alzheimers Dis. 2010;22(3): 1015-21. https://doi.org/10.3233/JAD-2010101185.

6. Razavi M, Tolea MI, Margrett J, Martin P, Oakland A, Tscholl DW, Ghods S, Mina M, Galvin JE. Comparison of 2 informant questionnaire screening tools for dementia and mild cognitive impairment: AD8 and IQCODE. Alzheimer Dis Assoc Disord. 2014;28(2):156-61. https://doi.org/10.1097/ WAD.0000000000000008.

7. Kivipelto M, Ngandu T, Laatikainen T, Winblad B, Soininen H, Tuomilehto J. Risk score for the prediction of dementia risk in 20 years among middle aged people: a longitudinal, population-based study. Lancet Neurol. 2006;5(9):735-41. https:// doi.org/10.1016/S1474-4422(06)70537-3.

8. Nasreddine ZS, Phillips NA, Bédirian V, Charbonneau S, Whitehead V, Collin I, Cummings JL, Chertkow H. The Montreal Cognitive Assessment, MoCA: a brief screening tool for mild cognitive impairment. J Am Geriatr Soc. 2005;53(4):695-9. https://doi.org/10.1111/j.1532-5415.2005.53221.x.

9. Folstein MF, Folstein SE, McHugh PR. "Mini-mental state": a practical method for grading the cognitive state of patients for the clinician. J Psychiatr Res. 1975;12(3):189-98. https://doi.org/10.1016/00223956(75)90026-6.

10. Yu P, Sun J, Wolz R, Stephenson D, Brewer J, Fox NC, Cole PE, Jack CR Jr, Hill DL, Schwarz AJ,
Coalition Against Major Diseases and the Alzheimer's Disease Neuroimaging Initiative. Operationalizing hippocampal volume as an enrichment biomarker for amnestic mild cognitive impairment trials: effect of algorithm, test-retest variability, and cut point on trial cost, duration, and sample size. Neurobiol Aging. 2014;35(4):808-18. https://doi. org/10.1016/j.neurobiolaging.2013.09.039 (Epub 2013 Oct 3. PMID: 24211008).

11. West T, Kirmess KM, Meyer MR, Holubasch MS, Knapik SS, Hu Y, Contois JH, Jackson EN, Harpstrite SE, Bateman RJ, Holtzman DM, Verghese PB, Fogelman I, Braunstein JB, Yarasheski KE. A bloodbased diagnostic test incorporating plasma A $\beta 42 / 40$ ratio, ApoE proteotype, and age accurately identifies brain amyloid status: findings from a multi cohort validity analysis. Mol Neurodegener. 2021;16(1):30. https://doi.org/10.1186/s13024-02100451-6.

12. Rabinovici GD, Gatsonis C, Apgar C, Chaudhary K, Gareen I, Hanna L, Hendrix J, Hillner BE, Olson C, Lesman-Segev OH, Romanoff J, Siegel BA, Whitmer RA, Carrillo MC. Association of amyloid positron emission tomography with subsequent change in clinical management among medicare beneficiaries with mild cognitive impairment or dementia. JAMA. 2019;321(13):1286-94. https://doi.org/10. 1001/jama.2019.2000.

13. Sabbagh MN, Lue LF, Fayard D, Shi J. Increasing precision of clinical diagnosis of Alzheimer's disease using a combined algorithm incorporating clinical and novel biomarker data. Neurol Ther. 2017;6(Suppl 1):83-95. https://doi.org/10.1007/ S40120-017-0069-5 (Epub 2017 Jul 21. PMID: 28733959; PMCID: PMC5520815). 